\title{
Vergütungssysteme in der integrierten Versorgung
}

\section{Auswahlkriterien, Kalkulation und Einsatzmöglichkeiten}

\begin{abstract}
Ziel dieser Analyse ist es, Kostenträgern eine Entscheidungshilfe bei der Auswahl und Ausgestaltung von projektspezifischen Vergütungssystemen in der integrierten Versorgung zu geben. Dabei werden Kriterien dargestellt, die für die Auswahl von Vergütungssystemen aus theoretischer Sicht relevant sind. Anschließend wird überprüft, inwieweit die vergütungstechnischen Voraussetzungen für eine disziplinenund sektorübergreifende Vergütung durch Einzelleistungen, Tagessätze, Komplexfallpauschalen und Kopfpauschalen in der Praxis bereits vorliegen.
\end{abstract}

\section{Sven Heinrich und Hans-Helmut König}

\section{Einleitung}

Seit dem Jahr 2000 haben Krankenkassen und Leistungserbringer die Möglichkeit, integrierte Versorgungsverträge nach §§ 140a-d des fünften Sozialgesetzbuches (SGB V) abzuschließen. Die integrierte Versorgung (IV) bezeichnet eine vertraglich vereinbarte, mehrere Leistungssektoren oder Fachdisziplinen umfassende Versorgungsform, mit dem Ziel, durch eine bessere sektor- und disziplinenübergreifende Kooperation und Koordination die Effektivität und Effizienz der medizinischen Versorgung in Deutschland zu erhöhen. ${ }^{1}$

Für die jährliche Finanzierung sind zunächst $1 \%$ der Vergütungen der Kassenärztlichen Vereinigungen und Krankenhäuser vorgesehen. Bisher scheinen davon jedoch im Jahr 2005 nur rund $50 \%$ ausgeschöpft zu werden. ${ }^{2}$ Ein Hinderungsgrund für die mangelnde Nutzung besteht in den Schwierigkeiten einer projektspezifischen Vergütung. Zwei Probleme treten dabei besonders in den Vordergrund. Zum einen ist dies die Auswahl eines Vergütungssystems für ein spezifisches Integrationsprojekt und zum anderen die Kalkulation der entsprechenden Vergütungshöhe. Es wird diskutiert, die Anschubfinanzierung auch 2007 fortzuführen. $^{3}$

\section{Verguitungssysteme in der integrierten Versorgung}

\subsection{Funktionen}

Vergütungssysteme erfüllen zwei wichtige Funktionen. Dies ist zum einen die Verteilungsfunktion und zum anderen die Steuerungsfunktion. Erstere wird durch einen

Dipl.-Kfm. Sven Heinrich, Prof. Dr. Hans-Helmut König, M.P. H. Stiftungsprofessur für Gesundheitsökonomie, Universität Leipzig
Ausgleich der gegenläufigen finanziellen Interessen des integrierten Versorgers und des Kostenträgers, z. B. der gesetzlichen Krankenversicherung (GKV) über die Vergütungshöhe bewerkstelligt. Letztere kann durch die Einbindung von Handlungsanreizen erfüllt werden. ${ }^{4}$ Sie sollen insbesondere die mit einer IV angestrebten Ziele unterstützen. Hier spielt vor allem die qualitätsorientierte Vergütung auf der Grundlage medizinischer Ergebnisindikatoren eine Rolle sowie die Setzung von Wirtschaftlichkeitsanreizen durch die Übertragung von ökonomischen Risiken.

\subsection{Charakteristika}

\subsubsection{Vergütungsverfahren}

Das Vergütungsverfahren beschreibt die institutionellen Rahmenbedingungen, in denen die Honorierung erfolgt. ${ }^{5}$ Aufgrund der direkten Vertragsgestaltung zwischen Kostenträger und Leistungserbringergemeinschaft kommt in der IV ein einstufiges Vergütungsverfahren zur Anwendung (Abbildung 1). Die Zwischenschaltung einer weiteren Institution wie der Kassenärztlichen Vereinigung entfällt.

Zwischen dem Kostenträger und dem Träger des Integrationsprojektes wird die äußere Vergütung verhandelt. Der Träger ist dadurch gekennzeichnet, dass er die Verantwortung für die Kooperation und Koordination des Projektes führt. Von der äußeren Vergütung, die die gesamten Leistungen des vertraglichen Integrationsversorgungsauftrages abdeckt, ist die innere Vergütung zu unterscheiden. Die innere Vergütung regelt die Honorierung der am Integrationsprojekt beteiligten Leistungserbringer entsprechend der innervertraglichen Festlegung. Dabei kann der Träger der IV gleichzeitig auch Leistungserbringer sein. Auch Leistungsanbieter, die nicht direkt 


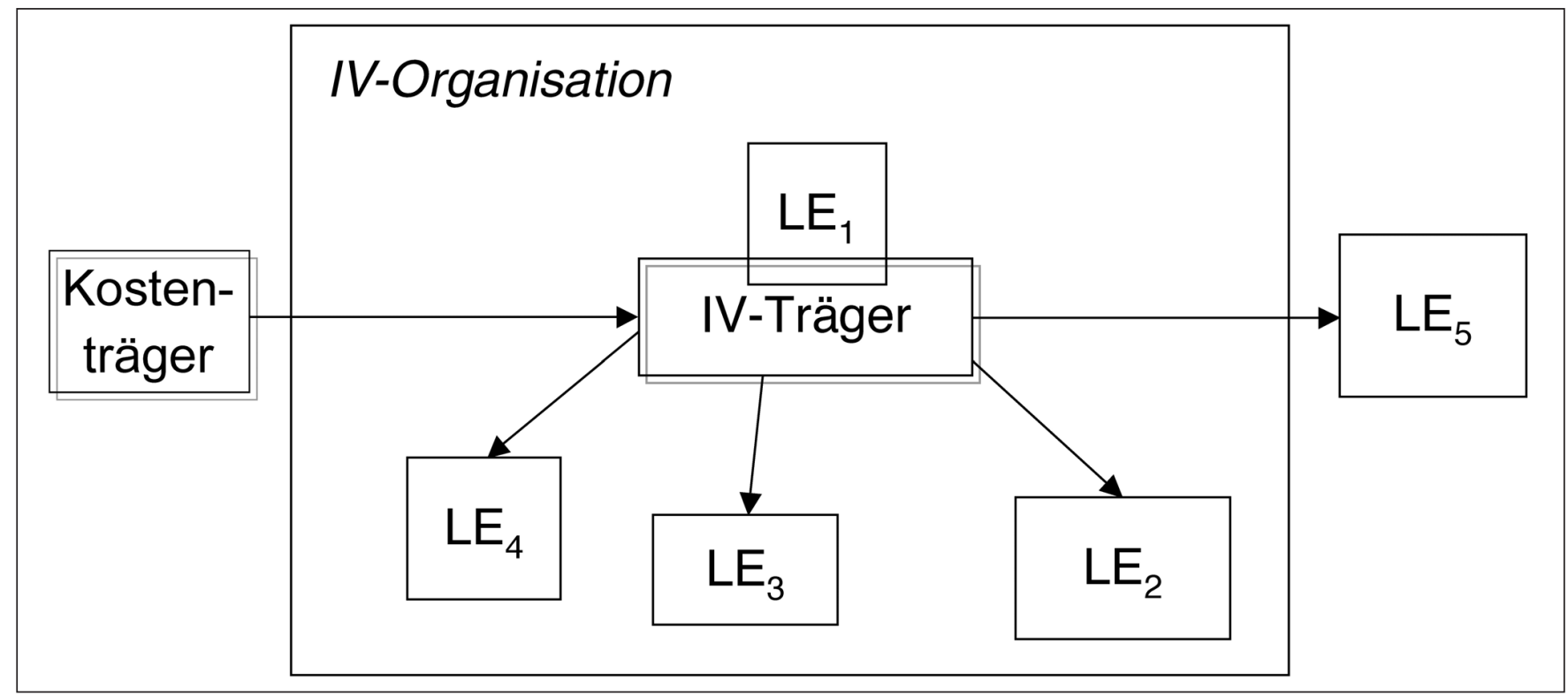

$\mathrm{LE}$, Leistungserbringer; $\rightarrow$ Vergütungsströme

Quelle: $\S \S 140 a-d$, SGB V idF v. 20.12.1988. BGL. I 1998: 2477 zuletzt geändert am 30.07.2004

in der integrierten Versorgungsorganisation integriert sind, werden aus der äußeren Vergütung des Trägers vergütet, soweit Versicherte von den am integrierten Versorgungsprojekt beteiligten Leistungserbringern im Rahmen des Versorgungsauftrages an jene überwiesen werden oder der Vertrag es verlangt. ${ }^{6}$

Die Bestimmung der äußeren Vergütung erfolgt zwischen dem integrierten Versorger und beispielsweise der GKV aufgrund eines wettbewerblichen Marktprozesses zum Beispiel in Form einer Ausschreibung. Dabei entstehen individuelle und selektive Verträge, die Bestimmungen zur Vergütungsform und zum Vergütungstarif enthalten.?

\subsubsection{Vergütungsform}

Die Vergütungsform wird durch den Maßstab bzw. die Maßstäbe, an die die Vergütung innerhalb einer Rechnungsperiode anknüpfen soll, definiert. ${ }^{8}$ Für die IV theoretisch geeignete Vergütungsformen werden in Tabelle 1 dargestellt. Andere mögliche äußere Vergütungsformen wie Faktoreinsatzmengen, vorgehaltene Betten sowie ein Perioden-Fixum scheiden aufgrund ihrer spezifischen Nachteile aus. Erstere setzt Anreize selbst Einzelleistungen ineffizient zu erbringen. ${ }^{9}$ Letztere steht aufgrund der Neubildungen der integrierten Versorgungsformen in Form von historisch gewachsenen Budgets in der Regel nicht zur Verfügung. Gehälter kommen nur in Betracht, wenn die Integrationsprojekte Eigeneinrichtungen der Kostenträger darstellen.
Integrationsverträge können ausdrücklich auch die Übernahme von Budgetverantwortung vorsehen. In diesem Zusammenhang wird in Deutschland auch der Begriff des kombinierten Budgets ${ }^{10}$ verwendet. Ein kombiniertes Budget ist dabei ein über bisher getrennte Sektoren gebildetes Gesamtbudget, welches eine Fixierung an den sektoralen Einzelbudgets überwindet. Im internationalen Sprachgebrauch ist dafür der Begriff der Kopfpauschale gebräuchlich und soll nachfolgend auch dafür verwendet werden.

Ein wesentliches Unterscheidungskriterium ist die mit der einzelnen Vergütungsform einhergehende Übertragung von ökonomischen Risiken auf den integrierten Versorger. Dabei werden prospektive (Kopf- und Komplexfallpauschalen) und retrospektive Vergütungssysteme

Tabelle 1: Geeignete Vergütungsformen für die integrierte Versorgung

\begin{tabular}{|l|l|}
\hline \multicolumn{1}{|c|}{ Vergütungsform } & \multicolumn{1}{c|}{ Begriff für das Vergütungssystem } \\
\hline Einzelleistungen & Einzelleistungsvergütung \\
\hline Pflege-/ Behandlungstage & Tagessätze \\
\hline $\begin{array}{l}\text { Behandlungsepisoden } \\
\text { Behandelte Patienten }\end{array}$ & Komplexfallpauschale \\
\hline Potentielle Patienten & Kopfpauschale (kombiniertes Budget) \\
\hline
\end{tabular}

Quelle: Eigene Darstellung 
(Tagessätze und Einzelleistungen) unterschieden. Bei prospektiven Vergütungssystemen wird die Vergütung im vornhinein festgelegt, womit das ökonomische Risiko des Versorgers steigt, bei retrospektiven wird die Leistungssituation rückblickend betrachtet, welche somit das ökonomische Risiko beim Kostenträger belassen. ${ }^{11}$

\subsubsection{Vergütungstarif}

Der Vergütungstarif wird bezogen auf das einzelne Integrationsprojekt ausgehandelt. Er beschreibt den funktionalen Zusammenhang zwischen der Vergütungshöhe P und dem der Vergütungsform zugrunde liegendem Maßstab bzw. den Maßstäben. ${ }^{12}$ Teilt sich das Gesamthonorar P des integrierten Versorgungsprojektes in Teilvergütungen auf, so ergibt sich jenes durch das Aufsummieren der einzelnen Teilvergütungen.

$$
\mathrm{P}=\mathrm{P}_{\text {Kopfpauschale }}+\mathrm{P}_{\text {Qualitätsanteil }}+\mathrm{P}_{\text {Leitlinienanteil }}
$$

Diese Formel verdeutlicht zum Beispiel eine Kopfpauschalvergütung verbunden mit einem ergebnisorientierten Vergütungsanteil und einem Anteil bezogen auf die Verwendung von Leitlinien. Die Höhe der Kopfpauschalteilvergütung ergibt sich durch die Multiplikation der Anzahl der potentiellen Patienten, beispielsweise differenziert nach Patientenklassen, mit dem zwischen den Vertragspartnern bestimmten Vergütungssatz. Der Vergütungssatz kann mit der Menge im Verlauf zunehmen, abnehmen oder konstant bleiben. ${ }^{13}$

\subsection{Theorie der Vergütung}

\subsubsection{Optimale Vergütungen}

Eine Vergütung ist aus Sicht des Kostenträgers optimal, wenn die in ihr wirkenden Anreize dazu führen, dass der integrierte Versorger entsprechend den Zielen des Kostenträgers handelt. Ein wesentliches Mittel, Steuerungswirkungen durch die Vergütung zu entfalten, ist die Übertragung eines Teils des ökonomischen Risikos auf den integrierten Versorger (prospektive Vergütungssysteme). Wie viel Risiko übertragen wird, hängt dabei von verschiedenen Kriterien ab. Insbesondere sind dies

- die Risikoeinstellung des Versorgers

- die Ziele des Kostenträgers, insbesondere hinsichtlich Qualität und Wirtschaftlichkeit

- die Möglichkeit der Risikoselektion ${ }^{14}$

\subsubsection{Risikoeinstellung des Versorgers}

Zur Ermittlung des optimalen Gesamtgrades der Risikoübertragung über die Vergütung ist zunächst die Risikoeinstellung des Versorgers und der Grad der Unsicherheit der Kosten von Bedeutung. Bezüglich der Risikoeinstellung werden risikofreudige, risikoneutrale und risikoaverse Einstellungen unterschieden. Risikoaverse Leistungserbringer sind bezüglich der Übernahme von Risiken negativ eingestellt. Risikoneutrale Leistungserbringer hingegen sind diesbezüglich indifferent. Risikoaverse Leistungserbringer kontrahieren deshalb bei der Setzung von Wirtschaftlichkeitsanreizen über prospektive Vergütungssysteme nur bei der zusätzlichen Zahlung einer Risikoprämie, was eine Erhöhung der Vergütung zur Folge hat. Durch die Risikoprämie wird die Übernahme des zusätzlichen Anteils am ökonomischen Risiko entgolten. Sowohl bei vermehrter Risikoaversion als auch bei einer größer werdenden Unsicherheit der Versorgungskosten sollte deshalb das Risiko verstärkt beim Kostenträger liegen, um so die Risikoprämie zu senken. Jedoch muss gleichzeitig eine Senkung des Anreizes zur Wirtschaftlichkeit in Kauf genommen werden. Sofern die Kosten sicher sind, ist die Risikoeinstellung von untergeordneter Bedeutung, da genau der Ressourceneinsatz honoriert werden kann, der auch entstehen wird. ${ }^{15}$

Bei der IV wird bei kleineren Projekten eher eine risikoaverse Einstellung vorherrschen, da relativ wenig Diversifikationsmöglichkeiten bezüglich anderer Tätigkeitsfelder bestehen, kaum Erfahrungen mit der IV vorhanden sind und zudem oft keine große Kapitaldecke besteht. Bei größeren Projekten, an denen Managementgesellschaften beteiligt sind, kann sich diese Aversion in Richtung Risikoneutralität abschwächen. Mit steigender Größe des IVProjekts und steigender Ähnlichkeit der behandelten Fälle kann die Unsicherheit der Kosten entsprechend zurückgehen, was sich positiv auf die zu zahlende Risikoprämie auswirken kann.

\subsubsection{Ziele des Kostenträgers}

Durch die Übertragung von ökonomischen Risiken auf das Integrationsprojekt entfalten sich auch Anreize bezogen auf die Wirtschaftlichkeit und die Qualität der Versorgung. Der Wirtschaftlichkeitsanreiz wirkt dann positiv, wenn es zu einer Reduzierung von nicht bedarfsgerechten Leistungsmengen und zu einer höheren Effizienz der Leistungserstellung bei gleichbleibender Qualität kommt.

Bei der Setzung von Wirtschaftlichkeitsanreizen spielt insbesondere die Beeinflussbarkeit der Kosten durch den integrierten Versorger eine entscheidende Rolle. Mit zunehmender Gestaltungsmöglichkeit sollte dabei auch die Verantwortung vermehrt auf den Versorger übergehen, da somit der Wirtschaftlichkeitsanreiz aufrechterhalten werden kann. Sofern die Sparanstrengungen kontrahierbar sind, entfällt die Notwendigkeit der Setzung eines Sparanreizes über das Vergütungssystem. ${ }^{16}$

Die Setzung eines Wirtschaftlichkeitsanreizes birgt aber auch immer die Gefahr, dass es zu einer Reduzierung der Versorgungsqualität kommt. Die Stärke des Anreizes muss deshalb mit den Auswirkungen auf die Qualität abgewogen werden. Zusätzliche vertragliche Regelungen wie 
Qualitätszirkel, Leitlinien oder ein Qualitätsmanagement können deshalb negative Wirkungen mit vermeiden. Wo möglich können die Einbindung von ergebnisorientierten Vergütungsbestandteilen als auch der Wettbewerb die Qualität der Versorgung zusätzlich erhöhen. Letzteres ist dann der Fall, wenn die Nachfrage nach Gesundheitsleistungen qualitätsabhängig ist. Spielt die Qualität keine Rolle, ist bei einem risikoneutralen Versorger die Übertragung der vollständigen Kostenverantwortung optimal. ${ }^{17}$

Die vermuteten Wirtschaftlichkeitsreserven ${ }^{18}$ im deutschen Gesundheitswesen sowie die Ziele, die mit der IV verfolgt werden sprechen, je nach Projekt, eher für eine prospektive Ausrichtung des Vergütungssystems, insbesondere auch deshalb, weil die Qualität in der IV zusätzlich über vertragliche Maßnahmen gefördert werden soll.

\subsubsection{Risikoselektion}

Aufgrund der Vergütung der zukünftig zu erwartenden durchschnittlichen Kosten pro Patient bei einer prospektiven Vergütung besteht für den integrierten Versorger ein Anreiz Patienten vermehrt zu behandeln, deren Kosten seiner Erwartung nach geringer als die prospektiv gezahlte Vergütung sind. Liegen die aus Sicht des Leistungser- bringers zu erwartenden Kosten über dem Vergütungssatz, bestehen Anreize diese Patienten zu vermeiden. ${ }^{19}$ Um diesen Risikoselektionsanreiz zu vermindern, bietet sich ausgehend von einer morbiditätsadjustierten Kopfbzw. einer Komplexfallpauschale eine weitere Verminderung des ökonomischen Risikos innerhalb des Vergütungssystems an. Mögliche Maßnahmen sind dabei eine zusätzliche Kostenübernahme durch den Kostenträger. Dies kann beispielsweise in Form der Übernahme von Hochkostenfällen geschehen. ${ }^{20}$

Da in der IV kein Kontrahierungszwang besteht, eröffnet sich auch in der IV die Möglichkeit zu einer Risikoselektion.

\subsection{Bestimmung eines projektspezifischen Vergütungssystems}

Wie viel ökonomisches Risiko auf ein spezifisches Integrationsprojekt übertragen wird, hängt von der Ausprägung der einzelnen Kriterien, bezogen auf das jeweilige IV-Projekt, ab. Die Entscheidungssituation des Kostenträgers kann mit Hilfe eines Entscheidungstableaus verdeutlicht werden (Abbildung 2). Für jedes einzelne Kriterium

\section{Abbildung 2: Entscheidungstableau}

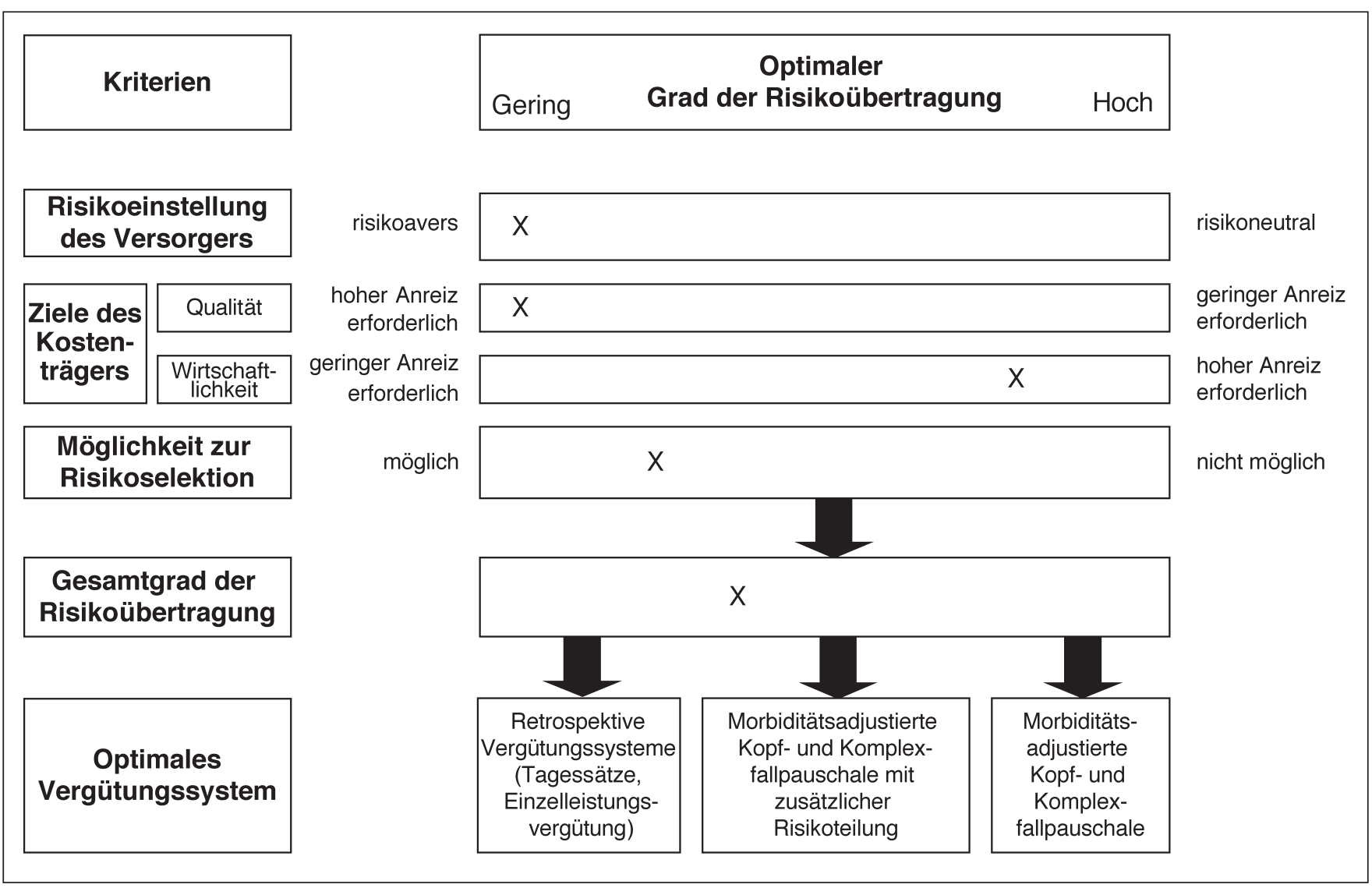

Quelle: Eigene Darstellung 
wird der optimale Grad der Risikoübertragung bestimmt und in das Entscheidungstableau eingetragen. Aus den einzelnen Kriterien lässt sich dann der Gesamtgrad der Risikoübertragung für das Vergütungssystem und ein entsprechendes Vergütungssystem bestimmen. Bei der Ermittlung des Gesamtgrades können prinzipiell zwei Strategien unterschieden werden. Entweder werden alle einzelnen optimalen Risikoübertragungsgrade gleichgewichtet oder es werden den einzelnen Kriterien unterschiedliche Gewichte zugeordnet.

In Abbildung 2 ist exemplarisch ein IV-Projekt mit unsicheren Kosten, einem risikoaversen IV-Träger, fehlender Überprüfbarkeit der Qualität der Behandlung und des Behandlungserfolges sowie Möglichkeiten zur Beeinflussung der Ressourcenverbräuche dargestellt. Bezogen auf die Risikoeinstellung ist es optimaler, wenn beispielsweise die annahmegemäß risikoneutrale GKV das ökonomische Risiko trägt, da sie somit die Risikoprämie senken kann. Selbiges gilt für die Qualität und den Anreiz zur Risikoselektion, ein geringeres Risiko kann hierbei Anreize setzen, zum einen die Behandlungsqualität nicht zu senken und zum anderen die Möglichkeit zur Risikoselektion nicht auszunutzen. In Konflikt geraten diese Kriterien mit dem Ziel der Wirtschaftlichkeit. Bei möglichen Kostenbeeinflussungspotentialen kann eine Übertragung des Risikos zu einer optimaleren Ressourcenverwendung führen.

Wenn - wie im dargestellten Beispiel - unterschiedliche optimale Risikoübertragungsgrade bezogen auf die einzelnen Kriterien bestehen, gibt es kein gesamtoptimales Vergütungssystem und es kann nur zwischen den verschiedenen Anreizen und der Höhe der Risikoprämie abgewogen werden. Die sich aus der Entscheidung für ein Vergütungssystem ergebenden negativen Konsequenzen, wie beispielsweise die Inkaufnahme einer geringeren Anstrengung zur Wirtschaftlichkeit, müssen dann durch weitere vertragliche Regelungen abgefedert werden. ${ }^{21}$

Die vermuteten Wirtschaftlichkeitsreserven im deutschen Gesundheitswesen sowie die Ziele, die mit der IV verfolgt werden, sprechen im Allgemeinen für die Übertragung von Risiken auf den Integrationsträger. Der vermuteten Risikoaversion, dem Qualitätssenkungsanreiz sowie der Risikoselektion kann mit einer auf das jeweilige Projekt zugeschnittenen zusätzlichen Risikoteilung begegnet werden. Die prospektive Vergütung kann dabei aus einer morbiditätsadjustierten Kopfpauschale oder Komplexfallpauschale bestehen. Dieser prospektive Teil kann sich wiederum in eine Grundvergütung ${ }^{22}$ und eine entsprechend angepasste Kopf- und Komplexfallpauschale aufteilen. Eine zusätzliche Risikoteilung kann beispielsweise mittels der Übernahme von Hochkostenfällen durch die GKV oder der Vereinbarung eines Maximalverlustes („stop loss“) erfolgen. In Anlehnung an die Regelungen im Krankenhaussektor können auch zusätzliche Beträge in Abhängigkeit von der Verweildauer gezahlt werden. Soll das Risiko aufgrund der Risikoaversion, Problemen bei der Qualitätssicherung oder einer ausgeprägten Risikoselektion ausschließlich bei der GKV liegen, bieten sich Tagessätze und Einzelleistungsvergütungen an.
Inwieweit das aus der Sicht des Kostenträgers bestimmte Vergütungssystem dann zum Einsatz kommt, hängt jedoch auch von der Verhandlungsmacht, dem Verhandlungsgeschick sowie der vergütungstechnischen Umsetzbarkeit ab. Letzteres bezieht sich vor allem auch auf die Frage der Kalkulation des entsprechenden Vergütungssystems. ${ }^{23}$

\section{Kalkulation und Einsatzmöglichkeiten von Vergütungssystemen}

\subsection{Kopfpauschalen}

\subsubsection{Kalkulation}

Bei der Kalkulation der Vergütungshöhe einer Kopfpauschale werden die zumeist für ein Jahr zu erwartenden durchschnittlichen zukünftigen Ausgaben pro eingeschriebenem Versicherten berechnet. Zur Prognose dieser Ausgaben stehen verschiedene Modelle zur Verfügung, die sich hinsichtlich ihrer Vorhersagekraft unterscheiden. Für die IV werden zur Kalkulation insbesondere die Modellfamilien ACG (Ambulatory Care Group bzw. Adjusted Clinical Group) / ADG (Ambulatory Diagnostic Group) und DCG (Diagnostic Cost Groups) / HCC (Hierarchical Coexisting Conditions bzw. Hierarchical Condition Categories) vorgeschlagen. ${ }^{24}$ Der Grund dafür ist der bereits erprobte Einsatz im amerikanischen Raum sowie der Umstand, dass sie nicht nur auf spezifische Versichertenkollektive hin entwickelt worden sind. Des Weiteren kann bei ersterer Modellfamilie z. T. eine Schätzung sowohl der ambulanten als auch der gesamten Leistungsausgaben erfolgen.

Eine erste Testung dieser Modelle mit innerdeutschen Daten erfolgte z. T. im Rahmen der Entwicklung des morbiditätsorientierten Risikostrukturausgleichs (RSA). ${ }^{25} \mathrm{Be}-$ zogen auf den RSA schnitt das relativ neue RxGroups + IPHCC (Inpatient Hierarchical Condition Categories) Modell aus der DCG / HCC Familie am besten ab. Dieses verwendet einen Regressionsansatz mit Krankenhausdiagnosen und ambulanten Arzneimittelverordnungen als Indikatoren für die Morbidität. Die zukünftigen Ausgaben für einen Versicherten ergeben sich aus Beträgen für das Alter, das Geschlecht und Zuschlägen für vorliegende Krankenhausdiagnosen sowie verordnete Arzneimittelwirkstoffe. Letztere werden sogenannten RxGroups zugeordnet, die wiederum in ARCs (Aggregated RX Categories) zusammengefaßt werden. Bei der Ermittlung der IPHCCs werden sämtliche stationäre Diagnosen eines Zeitraumes berücksichtigt. Grundlage für die Erhebung sind die Diagnosen nach ICD-9-CM, die zunächst in DxGroups und dann aufgrund klinischer und kostenmäßiger Aspekte weiter zu IPHCCs verdichtet werden. Die exemplarische Ermittlung einer jährlichen Kopfpauschale für eine 62jährige an Diabetes leidende Frau im RxGroups + IPHCC Modell ist in Tabelle 2 dargestellt. 
Tabelle 2: Kalkulationsbeispiel im RxGroups + IPHCC Modell (Sachleistungen ohne Zahnmedizin)

\begin{tabular}{|l|c|}
\hline Alter und Geschlecht & $517 €$ \\
\hline$+\quad$ RxGroup 115 (Insulinpräparate) & $2.406 €$ \\
\hline $\begin{array}{l}\text { HCC 019 (stationäre Behandlung wegen Diabetes } \\
\text { ohne oder mit nicht näher bezeichneten Komplika- } \\
\text { tionen) }\end{array}$ & $296 €$ \\
\hline Summe: & $3.219 €$ \\
\hline
\end{tabular}

Quelle: IGES, Lauterbach KW, Wasem J. Klassifikationsmodelle für Versicherte im Risikostrukturausgleich - Endbericht. 2004

Die über verschiedene Modelle ermittelten Kopfpauschalen je Risikoklasse lassen sich hinsichtlich der Güte der Kalkulation untersuchen. Bei der Auswahl von geeigneten Kalkulationsmodellen zur Kopfpauschalberechnung stehen zwei statistische Kriterien im Mittelpunkt. ${ }^{26}$ Zum einen ist dies der Anteil der Varianz an der Gesamtvarianz der Kosten, der durch die jeweiligen unabhängigen Variablen, z. B. Diagnosen, je Modell erklärt wird $\left(\mathrm{R}^{2}\right)$. Zum anderen ist dies die "Vorhersage-Relation" („predictive ratio“). Je größer $\mathrm{R}^{2}$ ist, desto geringer ist der
Risikoselektionsanreiz, der durch die, durch das jeweilige Modell bestimmte Kopfpauschale, ausgeht. $\mathrm{R}^{2}$ kann einen Wert zwischen 0 und 1 annehmen.

Der „predictive ratio“ ist definiert als das Verhältnis von vorhergesagten und tatsächlichen Kosten. Bei einem Wert von 1 stimmen die geschätzten mit den tatsächlichen Ausgaben überein. Diese Maßzahl bezieht sich dabei vorwiegend auf Teilkollektive von Versicherten. Insbesondere bei Gruppen mit einem besonderen Anreiz zur Risikoselektion ist deshalb ein Wert von nahezu 1 anzustreben.

Tabelle 3: Modellergebnisse des RSA (Sachleistungen ohne Zahnmedizin, ohne Beachtung der Trennung der Rechtskreise, der DMP Einschreibung und des Risikopools)

\begin{tabular}{|c|c|c|c|c|c|}
\hline \multirow[b]{2}{*}{ Gruppe } & \multirow[b]{2}{*}{ Modell } & \multirow[b]{2}{*}{ Typ } & \multirow[b]{2}{*}{$\mathbf{R}^{2}$} & \multicolumn{2}{|c|}{ „Predictive Ratio“ } \\
\hline & & & & Diabetes & $\begin{array}{c}\text { Koronare } \\
\text { Herzer- } \\
\text { krankung }\end{array}$ \\
\hline \multirow{2}{*}{ RSA } & \multirow{2}{*}{ RSA - Status Quo } & Prospektiv & $6 \%$ & 0,60 & 0,69 \\
\hline & & Zeitgleich & $7 \%$ & 0,58 & 0,62 \\
\hline \multirow{2}{*}{ ACG / ADG } & \multirow{2}{*}{ ACG (alle Leistungen) } & Prospektiv & $9 \%$ & 0,67 & 0,78 \\
\hline & & Zeitgleich & $14 \%$ & 0,68 & 0,73 \\
\hline \multirow{4}{*}{ DCG / HCC } & \multirow{2}{*}{$\mathrm{HCC}$} & Prospektiv & $15 \%$ & 0,87 & 0,86 \\
\hline & & Zeitgleich & $26 \%$ & 0,87 & 0,83 \\
\hline & \multirow{2}{*}{ RxGroups + IPHCC } & Prospektiv & $24 \%$ & 0,91 & 0,99 \\
\hline & & Zeitgleich & $48 \%$ & 0,93 & 1,01 \\
\hline
\end{tabular}

Quelle: IGES, Lauterbach KW, Wasem J. Klassifikationsmodelle für Versicherte im Risikostrukturausgleich - Endbericht. 2004 
Leistungen einschließende Versorgung (ambulante Vollversorgung) kalkuliert werden. Die oben beschriebenen Kennwerte kommen hier ebenso zum Tragen. ${ }^{28}$

\subsubsection{Einsatzmöglichkeiten}

Eine Vergütung über Kopfpauschalen kommt vor allem für eine ambulante Vollversorgung oder sektorübergreifende Vollversorgung in Betracht. Kopfpauschalen können jedoch auch für einzelne Indikationen erwogen werden, insbesondere sektorübergreifende Leistungskomplexe im Rahmen von Disease-Management-Programmen für chronische Erkrankungen scheinen hierfür geeignet.

Problematisch für die IV in Deutschland ist der Umstand, dass noch kein Modell hinsichtlich der ambulanten oder sektorübergreifenden Vollversorgung explizit auf eine Eignung für die IV in Deutschland untersucht wurde. Erste statistische Kennwerte verschiedener Modelle, die für eine sektorübergreifende Vollversorgung in Frage kommen könnten, können den Berechnungen für den morbiditätsadjustierten RSA entnommen werden. Inwieweit das RxGroups + IPHCC Modell jedoch trotz der guten statistischen Kennwerte das Modell der Wahl für eine sektorübergreifende Vollversorgung ist, bleibt offen, da bei der generellen Entscheidung für ein Modell andere Kriterien bzw. Kriteriengewichtungen als beim RSA Anwendung finden müssen. Beispielsweise spielt bei der IV die Kontrollierbarkeit der Inputdaten eine größere Rolle, da sie von den zu vergütenden Leistungserbringern selbst erhoben werden. ${ }^{29}$ Eine kurzfristige Umsetzbarkeit einer sektorübergreifenden Vollversorgungsvergütung scheint somit noch nicht möglich (Tabelle 4). Ein Einsatz der derzeit geltenden RSA Pauschalen in der IV-Vollversorgung bedeutet für die Leistungserbringer ein zu hohes Risiko und scheidet deshalb aus. Die Ermittlung der Kennwerte verschiedener Modelle, die auch für eine Vergütung von IV-Projekten einer ambulanten Vollversorgung in Frage kommen könnten, wird momentan im Zuge der Einführung der Regelleistungsvolumina in der ambulanten Versorgung forciert und diskutiert. ${ }^{30}$ Eine Übernahme amerikanischer Kostengewichte ist nicht möglich, da die im amerikanischen Kontext den jeweiligen Diagnosen zugeordneten Kosten aufgrund anderer Schnittstellen nicht notwendigerweise denen in Deutschland entsprechen müssen. ${ }^{31}$ Eine Übernahme der momentan an die Kassenärztlichen Vereinigungen gezahlten Kopfpauschalen als Grundlage für die Vergütung einer ambulanten Vollversorgung ist aufgrund der Unabhängigkeit von Alter, Geschlecht und Morbidität wegen des hohen Risikos nicht sinnvoll. ${ }^{32}$

\subsection{Komplexfallpauschale}

\subsubsection{Kalkulation}

Die Vergütungsregelung bei einem Versorgungsprojekt mit Komplexfallpauschalen bezieht sich auf die Vergütung von einzelnen Fällen über sektorale und/oder diszi- plinäre Grenzen hinweg. Grundlage dafür sind auch hier Patientenklassifikationssysteme, die die Patienten in klinisch definierte Gruppen mit ähnlichen Behandlungskosten einteilen. ${ }^{33}$ Bisher bestehen solche Systeme vorwiegend für den stationären Bereich (DRG - Diagnosis Related Group). Eine Vergütung über Fallpauschalen in anderen Sektoren, wie der Rehabilitation und deren anschließenden Verbindung, setzt dabei z.T. die Entwicklung neuer Klassifikationssysteme voraus. ${ }^{34}$ Inwieweit für einen sektorübergreifenden Leistungskomplex dann eine weitere Falldifferenzierung vorgenommen wird, hängt von der Kostenhomogenität des jeweiligen Versorgungsauftrages ab. Eine Orientierung am stationären Sektor bietet sich jedoch an.

Die differenzierte Fallkostenbestimmung kann dann in Anlehnung an die Kostenermittlung der DRGs über eine Kostenarten-, Kostenstellen- und Kostenträgerrechnung erfolgen ${ }^{35}$, die entsprechend um die hinzukommenden Kostenarten und Kostenstellen, z. B. der Rehabilitation, erweitert werden. Nachrangig kann auch eine Kalkulation von Sollkosten aufgrund optimaler Behandlungspfade ${ }^{36}$ oder ein prozentualer Zu- oder Abschlag auf die DRG erwogen werden. Bei der Ersetzung von stationären durch ambulante Leistungen ist die DRG bei gleichbleibender Leistung somit entsprechend abzusenken, bei der Integration von Rehabilitationsleistungen in der Regel zu erhöhen.

Bei der Kalkulation der Vergütungshöhe mit Einbezug des stationären Sektors ist jedoch zu beachten, dass über die IV-Vergütung nur Leistungen honoriert werden dürfen, die über die im Gesamtbetrag enthaltenen Leistungen hinausgehen. ${ }^{37}$

\subsubsection{Einsatzmöglichkeiten}

Aus institutioneller Sicht eignet sich die Vergütung über Komplexfallpauschalen insbesondere dort, wo eine Einbindung von DRGs möglich ist. Hierbei kommen vor allem die Verbindungen zum Rehabilitationssektor und zum ambulanten Sektor in Frage. Bei letzterem können stationäre Leistungen durch ambulante ersetzt werden..$^{38}$ Auch interdisziplinäre Projekte innerhalb des stationären ${ }^{39}$ und ambulanten Sektors können über eine Komplexfallpauschale vergütet werden. Im ambulanten Sektor allein ist vor allem das ambulante Operieren ${ }^{40} \mathrm{zu}$ nennen (Tabelle 4).

Jedoch ist der Einsatz von Komplexfallpauschalen im Rehabilitationsbereich umstritten. ${ }^{41}$ Positiv ist zu werten, dass über eine Komplexfallpauschale Anreize zur besseren Kooperation und Koordination, insbesondere beim Übergang in den Rehabilitationssektor, gesetzt werden. Dies kann sich in einer Reduzierung der Gesamtbehandlungsdauer, insbesondere auch durch die Vermeidung von Wartezeiten, niederschlagen. Für die Indikationsbereiche Orthopädie, Kardiologie und Neurologie wurden entsprechende Rehabilitationsfallpauschalen bereits entwickelt. ${ }^{42}$ Anzumerken ist jedoch, dass sich die grundlegenden Behandlungsziele zwischen Akut- und Rehabilitationsbehandlung unterscheiden und somit auch die Optimalität einer Komplexfallpauschale. Aufgrund des relativ konstanten Kostenverlaufs in der Rehabilitation 
kann es zu einer verstärkten Qualitätsminderung kommen. Ebenso ist im Gegensatz zur Akutklinik eine Reduzierung der Behandlungsdauer in der medizinischen Rehabilitation nur begrenzt möglich, da übende und trainierende Aspekte im Mittelpunkt stehen. Der Sachverständigenrat für die Konzertierte Aktion im Gesundheitswesen plädiert deshalb für ein separates Vergütungssystem. ${ }^{43}$ Dieses beinhaltet einrichtungsübergreifende, nach dem Rehabilitationsbedarf differenzierte Tagessätze auf der Grundlage eines Patientenklassifikationssystems. Des Weiteren soll die festgelegte Rehabilitationshöchstdauer auf der Grundlage von Behandlungsleitlinien und Therapieplänen flexibilisiert werden. In Anlehnung an unterschiedliche Vergütungsmöglichkeiten für die Frührehabilitation in Akutkliniken ist deshalb auch in der IV eine Kombination aus einer Fallpauschale mit einem Tagessatz vorstellbar. ${ }^{44}$ Dem Anreiz zur vorzeitigen Verlegung in den über Tagessätze abgedeckten Leistungsbereich kann durch eine Mindestverweildauer in der Akutklinik entgegengewirkt werden.

\subsection{Tagessätze}

\subsubsection{Kalkulation}

Tagessätze bestimmen den durchschnittlichen Ressourcenverbrauch pro Tag und können aufgrund historisch gewachsener Kostenstrukturen oder prospektiv ermittelt werden. ${ }^{45}$ Die Ermittlung von prospektiven Tagessätzen kann ähnlich wie bei der Komplexfallpauschale über eine entsprechende Kostenrechnung auf der Grundlage des Integrationsprojektes erfolgen. Kostenträger ist dann der jeweilige Behandlungstag. ${ }^{46}$ Bei differenzierten Tagessät- zen bezüglich verschiedener Fälle ist, wie bei der Komplexfallpauschale, ein Patientenklassifikationssystem als Grundlage notwendig. ${ }^{47}$

\subsubsection{Einsatzmöglichkeiten}

In der IV können Tagessätze prinzipiell sowohl im stationären Akutsektor als auch in der ambulanten und stationären Rehabilitation zur Anwendung kommen. Dabei kann auf die derzeit geltenden Tagessätze im Rehabilitationssektor bzw. bei Projekten im psychosozialen Bereich auf die Tagessätze der Psychiatrie, Psychosomatik sowie der psychotherapeutischen Medizin zurückgegriffen werden. ${ }^{48}$ Inwieweit diese jedoch vor allem historisch gewachsenen Tagessätze dem Ressourcenverbrauch einer in der IV zu behandelnden differenzierten Patientengruppe entsprechen, bleibt offen. Auch hier fehlen notwendige Patientenklassifikationssysteme (Tabelle 4). ${ }^{49}$

\subsection{Einzelleistungsvergütung}

\subsubsection{Kalkulation}

Bei der Kalkulation von Einzelleistungen wird der durchschnittliche Ressourcenverbrauch pro einzelner Leistung bestimmt. Für die IV ist vor allem die Methodik des neuen EBM 2000 Plus von Bedeutung. ${ }^{50}$ Sie geht im wesentlichen auf die RBRVS (Resource-Based Relative Value Scale) zurück. ${ }^{51}$ Der Wert einer Einzelleistung im neuen EBM 2000 Plus ergibt sich dabei vor allem aus zwei Hauptkostenkomponenten, die über Kostenträgerrech-

Tabelle 4: Gegenüberstellung der Vergütungsmöglichkeiten

\begin{tabular}{|c|c|c|c|c|}
\hline & \multicolumn{2}{|c|}{ Prospektive Vergütung } & \multicolumn{2}{|c|}{ Retrospektive Vergütung } \\
\hline Vergütungsform & $\begin{array}{l}\text { Zeitraumbezogene } \\
\text { Kopfpauschale }\end{array}$ & Komplexfallpauschale & Tagessätze & Einzelleistungen \\
\hline $\begin{array}{l}\text { Kurzfristige } \\
\text { Umsetzbarkeit }\end{array}$ & $\begin{array}{l}\text { nein, nur mit hohem } \\
\text { Risiko auf der } \\
\text { Grundlage des derzeit } \\
\text { geltenden RSA }\end{array}$ & $\begin{array}{l}\text { ja, vor allem: } \\
\text { sektorübergreifender } \\
\text { Leistungskomplex Akut- } \\
\text { und Rehabilitationsklinik } \\
\text { mit evtl. ambulantem } \\
\text { Sektor sowie } \\
\text { sektorübergreifender } \\
\text { Leistungskomplex } \\
\text { stationärer mit ambulantem } \\
\text { Sektor } \\
\text { interdisziplinäre IV-Projekte } \\
\text { im stationären sowie z. T. im } \\
\text { ambulanten Bereich }\end{array}$ & $\begin{array}{l}\text { ja, vor allem: } \\
\text { - } \quad \text { stationärer Sektor } \\
\text { und Rehabilitations- } \\
\text { bereich } \\
\text { - } \quad \text { interdisziplinäre } \\
\text { Projekte im } \\
\text { stationären Sektor }\end{array}$ & $\begin{array}{l}\text { ja, vor allem: } \\
\text { - } \text { interdisziplinäre } \\
\text { Projekte im ambulanten } \\
\text { Bereich } \\
\text { - } \quad \text { ambulante } \\
\text { Vollversorgung }\end{array}$ \\
\hline \multirow{2}{*}{ Probleme } & \multicolumn{3}{|c|}{ Auswahl bzw. Entwicklung von Klassifikationssystemen } & $\begin{array}{c}\text { Definition von } \\
\text { Einzelleistungen }\end{array}$ \\
\hline & \multicolumn{4}{|c|}{ Bewertung der Leistungen } \\
\hline
\end{tabular}

Quelle: Eigene Darstellung 
nungen bestimmt werden. Dies ist zum einen die Arbeitsleistung und zum anderen die technische Leistung. Die technische Leistung beinhaltet Leistungen wie beispielsweise den Einsatz eines Ultraschallgerätes. Die Opportunitätskosten für die Fort- und Weiterbildung finden Eingang in den Kostensatz der Arbeitsleistung. Durch diese Methodik wird ein relativer Wert für jede Einzelleistung ermittelt, der anschließend in Punkten ausgedrückt wird. Ein Punkt wird dann mit einem monetären Wert belegt. ${ }^{52}$ Dieser monetäre Wert kann in der IV unabhängig von den für die Regelversorgung geltenden Punktwerten festgelegt werden, so dass eine Kostendeckung gewährleistet ist.

\subsubsection{Einsatzmöglichkeiten}

Bei Projekten im ambulanten Bereich, evtl. mit Einbindung der Arznei-, Heil- und Hilfsmittel, kann auf bereits bestehende Leistungsdefinitionen und Bewertungen zurückgegriffen werden. Prinzipiell sind aber auch andere Leistungsdefinitionen und Punktwerte als in der Regelversorgung möglich. Aufgrund der fehlenden Einzelleistungsdefinitionen und Bewertungen im stationären Sektor sowie im Rehabilitationsbereich wird kurz- bis mittelfristig eine Einzelleistungsvergütung hier nicht umsetzbar sein. Unabhängig von dem Einsatz als retrospektives Vergütungssystem, welches sämtliche Leistungen abdeckt, kann die Einzelleistungsvergütung auch als Ergänzung zu prospektiven Vergütungssystemen im Rahmen eines modularen Aufbaus ${ }^{53}$ Verwendung finden (Tabelle 4).

\section{Empirische Ergebnisse zum Einsatz von Vergütungs- systemen in der integrierten Versorgung}

Genaue empirische Untersuchungen zum tatsächlichen Einsatz von verschiedenen Vergütungssystemen und ihrer Ausgestaltung sind für Deutschland derzeit noch nicht vorhanden. Es können nur Hypothesen auf der Grundlage der geschlossenen Verträge, beispielsweise über die freiwilligen Meldungen von Verträgen an die gemeinsame Registrierungsstelle, der IV Datenbank Deutschland (Hildebrandt GesundheitsConsult) ${ }^{54}$ oder aufgrund von veröffentlichten Projektdaten in Zeitschriften aufgestellt werden.

Ein Fokus scheint aber mit auf Komplexfallpauschalen zu liegen, da diese vor dem Hintergrund des Know-hows von Krankenhäusern und der Einführung von DRGs eher umsetzbar sind..$^{55}$

\section{Fazit}

Im Mittelpunkt stand die Frage, wie spezifische Integrationsprojekte aus Sicht eines Kostenträgers optimal zu vergüten sind und bei welchen sektor- und / oder disziplinenübergreifenden Vergütungssystemen die Vergütungshöhe in Deutschland bereits kalkuliert werden kann. Ein Vergütungssystem ist dabei selten hinsichtlich aller Kriterien optimal. Mit Hilfe eines Entscheidungstableaus kön- nen die verschiedenen Anreize, bezogen auf die Risikoeinstellung, die Ziele des Kostenträgers und der Möglichkeit zur Risikoselektion für ein spezifisches Projekt analysiert werden. Inwieweit ein Vergütungssystem dann praktisch eingesetzt wird, hängt jedoch neben der Verhandlungsmacht und dem Verhandlungsgeschick auch von der Umsetzbarkeit und somit dem Vorhandensein von Leistungsdefinitionen und Bewertungsansätzen ab.

Hinsichtlich der Berechnung der Vergütungshöhe einer Kopfpauschale liegen erstmalig über die Weiterentwicklung des RSA gewonnene Kennwerte verschiedener morbiditätsadjustierter Modelle mit innerdeutschen Daten vor. Ebenso werden im Zuge der Umstellung auf eine morbiditätsorientierte ambulante Vergütung Modelle zur Kopfpauschalberechnung getestet. Jedoch fehlt eine Prüfung der Modelle bezogen auf den Einsatz in der IV. Die Komplexfallpauschale bietet bei dem Vorhandensein von betriebswirtschaftlichem Know-how zur Kalkulation der Vergütungshöhe gute Chancen insbesondere auch für eine sektorübergreifende Vergütung. Problematisch ist jedoch das Fehlen von Klassifikationssystemen, insbesondere für den ambulanten sowie z. T. für den Rehabilitationsbereich. Letzteres wirkt sich auch negativ auf die Kalkulation von differenzierten Tagessätzen aus. Eine Einzelleistungsvergütungsvereinbarung kann vor allem für interdisziplinäre Projekte im ambulanten Bereich Anwendung finden. ${ }^{56}$ Für eine sektorübergreifende Vergütung ist sie jedoch kurzfristig kaum einsetzbar, da außerhalb der vertragsärztlichen Versorgung die dazu notwendigen Einzelleistungsdefinitionen fehlen.

Die praktische Umsetzbarkeit scheint somit derzeit vorrangig für interdisziplinäre Projekte in einem Sektor mit dem jeweiligen Vergütungssystem der Regelversorgung möglich. Für die Nutzung der Chancen, die mit einer Integration von sektorübergreifenden Versorgungsprozessen einhergehen, ist jedoch der weitere Ausbau der vorhandenen Grundlagen für eine sektorübergreifende Kalkulation der Vergütungshöhe notwendige Voraussetzung. Im Zuge der Einführung des morbiditätsorientierten RSA und einer morbiditätsorientierten ambulanten Vergütung eröffnen sich auch für die Vergütung von Integrationsleistungen neue Perspektiven und Chancen. Von einer auf das Einzelprojekt abgestimmten Vergütung, die die mit der Integrationsversorgung verfolgten Ziele unterstützt, können dann auch weitere Impulse für die Weiterentwicklung der Integrationsversorgung an sich und somit auch des deutschen Gesundheitswesens ausgehen.

\section{Fußnoten}

1 \& 140a Absatz I Satz 1 SGB V idF v. 20.12.1988. BGBl. I 1998: 2477 zuletzt geändert am 30.07.2004 und SVR KAiG. Gutachten 2003 Finanzierung, Nutzerorientierung, Qualität Bd I und II. Bundestagsdrucksache 15/530, 2003

2 BQS Bundesgeschäftsstelle Qualitätssicherung gGmbH. Informationen 3. Quartal 2005. www.bqs-register140d.de. 2005, Henze M, Pöppl SJ. Koproduktion zwischen den Sektoren - Integrationsnetzwerke sind für den künftigen Wettbewerb gut aufgestellt. Ku - Sonderheft: Integrierte Versorgung 2004; 73: 18-21 und Strang A, Schulze S. Integrierte 
Versorgung - Mit neuen Partnern über alte Grenzen. Gesundheit und Gesellschaft 2004; 7: 32-37

3 Knieps F. BMGS auf Einladungskonferenz - Die Zukunft ist chronisch. Das Chronic Care Model in der Primärmedizin, Frankfurt/Main 2005

4 Wiechmann M. Managed Care - Grundlagen, internationale Erfahrungen und Umsetzung im deutschen Gesundheitssystem. Wiesbaden: Dt. Univ.-Verl., 2003: 66

5 Schulenburg JM Graf von der. Systeme der Honorierung frei praktizierender Ärzte und ihre Allokationswirkungen. Tübingen: Mohr, 198

6 § 140c SGB V. idF v. 20.12.1988. BGBl. I 1998: 2477 zuletzt geändert am 30.07.2004

7 Amelung VE, Schumacher H. Managed Care - Neue Wege im Gesundheitsmanagement. 3. Aufl. Wiesbaden: Gabler, 2004: 28-143 und Ebsen I. Kartell- und vergaberechtliche Aspekte des vertraglichen Handelns der Krankenkassen. Die Krankenversicherung 2004; 56: 95-100

8 Breyer F, Zweifel P, Kifmann M. Gesundheitsökonomik. 5. Aufl. Berlin u. a.: Springer, 2005

9 Breyer F, Zweifel P, Gesundheitsökonomie. 3. Aufl. Berlin u.a.: Springer, 1999

10 Schönbach KH. Kombinierte Budgets. Die BKK 1994; 82: 390-395

11 Breyer F, Zweifel P, Kifmann M. (2005) a.a.O.

12 Ebenda

13 Ebenda und Eichhorn P, Seelos HJ, Schulenburg JM Graf von der. Krankenhausmanagement. München Jena: Urban und Fischer, 2000: 551

14 Breyer F, Zweifel P, Kifmann M. (2005) a.a.O., Pratt W, Zeckhauser RJ. Principals and Agents: The Structure of Business. Boston Mass: Harvard Business School Pr., 1991, Arrow K. Uncertainty and the welfare economics of medical care. The American Economic Review 1963; 53 (5): 851-883, Spremann K. Asymmetrische Informationen. Zeitschrift für Betriebswirtschaft 1990; 60: 561-568, Milgrom P, Roberts J. Economics, Organization \& Management. New Jersey: Prentice Hall, 1992

15 Ebenda und Erlei M, Leschke M, Sauerland D. Neue Institutionenökonomik. Stuttgart: Schäffer-Poeschel, 1999

16 Breyer F, Zweifel P, Kifmann M. (2005) a.a. O.

17 Ebenda und Buchner F, Ryll A, Wasem J. Periodenbezogene Vergütungssysteme: Die risikoadjustierte Kopfpauschalvergütung. In: Wille E (Hrsg). Anreizkompatible Vergütungssysteme im Gesundheitswesen. Baden-Baden: Nomos-Verlagsgesellschaft, 2002: 63-95, Stade U. Anreizwirkungen in den Beziehungen zwischen Arzt, Patient und Krankenkasse. Hamburg: Kovac, 2004 und Chalkley M, Malcomson JM. Government Purchasing of Health Services. In: Culyer AJ, Newhouse, JP (Hrsg). Handbook of Health Economics, Volume 1A. Amsterdam u. a.: Elsevier, 2000: 848-890

18 SVR KAiG, Gutachten 2000/2001 Bedarfsgerechtigkeit und Wirtschaftlichkeit Band III. Bundestagsdrucksache 14/6871, 2001

19 Ellis RP. Creaming, skimping, dumping: provider competition on the intensive and extensive margins. Journal of Health Economics 1998; 17: 537-555

20 Breyer F, Zweifel P, Kifmann M. (2005) a. a. O.

21 Ebenda

22 Sachverständigenrat zur Begutachtung der gesamtwirtschaftlichen Entwicklung. Zwanzig Punkte für Beschäftigung und Wachstum Jahresgutachten 2002/2003. Stuttgart: Metzler-Poeschel, 2002 und Krauth C u.a.. Zur Umsetzung ergebnisorientierter Vergütungselemente in der ambulanten Versorgung. Arbeit und Sozialpolitik 1998; 52 Heft 11/12: 10-22

23 Breyer F, Zweifel P. (1999) a. a.O.

24 Buchner F, Ryll A, Wasem J. (2002) a.a. O, Schräder WF, Ryll A. Pauschalierende Vergütungssysteme in der integrierten Versorgung. in: Tophoven C, Lieschke L (Hrsg). Integrierte Versorgung - Entwicklungsperspektiven für Praxisnetze. Köln: Dt.-Ärzte-Verlag, 2001: 130-169 und Weiner JP u.a.. Development and application of a populationoriented measure of ambulatory care case-mix. Medical Care 1991; 29: $452-472$

25 IGES, Lauterbach KW, Wasem J. Klassifikationsmodelle für Versicherte im Risikostrukturausgleich - Endbericht. 2004

26 Buchner F, Ryll A, Wasem J. (2002) a.a.O. und IGES, Lauterbach KW, Wasem J. (2004) a.a.O.

27 IGES, Lauterbach KW, Wasem J. (2004) a.a. O

28 Maydell B von, Schulte C. Möglichkeiten zur Justierung von Patientenklassifikationssystemen für die ambulante Vergütung eine Analyse mit Hilfe der Clinical Risk Groups. Gesundheits- und Sozialpolitik 2005; 59 Heft 1/2: 28-35 und Reschke P et al. Methoden der Morbiditätsadjustierung. Gesundheits- und Sozialpolitik 2005; 59 Heft 1/2: $10-19$
29 IGES, Lauterbach KW, Wasem J. Klassifikationsmodelle für Versicherte im Risikostrukturausgleich - Zwischenbericht. 2002

30 Maydell B von, Schulte C. (2005) a.a.O., Reschke P et al. (2005) a.a.O. und Andersen HH, Mühlbacher AC. Morbidität, Bedarf und Vergütung - Methodische Grundlagen für Analysen über Zusammenhänge in der ambulanten Versorgung - Das Beispiel morbiditätsorientierter, regionalisierter Prognosen des Ärztebedarfs. Gesundheits- und Sozialpolitik 2005; 59 Heft 5/6: 20-30

31 Buchner F, Ryll A, Wasem J. (2002) a.a.O.

32 Sachverständigenrat zur Begutachtung der gesamtwirtschaftlichen Entwicklung (2002) a.a.O.

33 Fischer W. Grundzüge von DRG-Systemen. In: Arnold M, Lietsch M, Schellschmidt H (Hrsg). Krankenhaus-Report 2000 - Schwerpunkt: Vergütungsreform mit DRGs. Stuttgart: Schattauer, 2001: 13-31

34 SVR KAiG. Gutachten 2003 Finanzierung, Nutzerorientierung, Qualität Bd I und II. Bundestagsdrucksache 15/530, 2003 und Haaf HG, Volke E, Schliehe F. Neue Vergütungs- und Versorgungsformen und ihre Auswirkungen auf die Rehabilitation. Die Rehabilitation 2004; 43: 312-324

35 DKG, GKV, PKV. Kalkulation von Fallkosten - Handbuch zur Anwendung in Krankenhäusern, Version 2.0. Düsseldorf: Dt. KrankenhausVerl.-Ges., 2002

36 Amelung VE, Schumacher H. (2004) a.a.O.

$37 \S 140 d$ Absatz IV Satz 1 SGB V idF v. 20.12.1988. BGB1. I 1998: 2477 zuletzt geändert am 30.07.2004

38 Keller J. Das ist integra. Integra - Das Magazin für Ärzte, Patienten und Besucher 2004; 14

39 DKG. GKV Modernisierungsgesetz: Neue Versorgungsformen im Krankenhaus. Düsseldorf: DKVmbH, 2004: 8

40 Hildebrandt GesundheitsConsult GmbH. Integrierte Versorgung Stand der Dinge: Die aktuelle Vertragslandschaft - Erfahrungen und Probleme. Ku - Sonderheft: Integrierte Versorgung 2004; 73: 5

41 SVR KAiG. (2003) a.a.O. und Haaf HG, Volke E, Schliehe F. (2004) a.a.O.

42 SVR KAiG. Sondergutachten 1997 Gesundheitswesen in Deutschland, Kostenfaktor und Zukunftsbranche, Band II: Finanzierung und Vergütung. Baden-Baden: Nomos-Verlagsgesellschaft, 1998 und Nowy R. Ergebnisorientierte fallbasierte Vergütung. Bayreuth: PCO, 2002

43 SVR KAiG. (2003) a.a.O.

44 Maylath E. DRGs in der psychiatrischen Krankenhausfinanzierung am Beispiel Ungarns. Ein Modell für Deutschland? Das Gesundheitswesen 2000; 62: 633-645

45 Neubauer G. Formen der Vergütung von Krankenhäusern und deren Weiterentwicklung. In: Braun GE (Hrsg). Handbuch Krankenhausmanagement. Stuttgart: Schäffer-Poeschel, 1999: 27

46 Endt, HJ von, Gutt HB, Weber-Sieb J. Die Ermittlung leistungsgerechter Pflegesätze (Entgelte) nach § 93 BSHG n.F. und § 84ff. SGB XI auf der Basis des individuellen Pflege- und Betreuungsaufwandes und der einrichtungsbezogenen Leistungskosten. Pflegezeitschrift 1995; 48: 2-11

47 Haaf HG. Gesundheitsökonomische Analyse der Vergütung mit Fallpauschalen in der medizinischen Rehabilitation. Die Rehabilitation 2002; 41: 14-30

48 Tuschen KH, Trefz U. Krankenhausentgeltgesetz - Kommentar. Stuttgart: Kohlhammer, 2004

49 SVR KAiG. (2003) a.a.O.

50 Munte A, Popp W. Zukünftige gebietsärztliche Vergütung. In: Albring M, Wille E (Hrsg.). Qualitätsorientierte Vergütungssysteme in der ambulanten und stationären Behandlung. Frankfurt am Main u. a.: Lang, 2001, 107-127

51 Hsiao WC et al. An overview of the development and refinement of the Resource-Based Relative Value Scale. Medical Care 1992; Heft 11 - Supplement: 1-12

52 Munte A, Popp (2001) a.a.O. und Hsiao WC et al. Results and impacts of the Resource-Based Relative Value Scale. Medical Care 1992; Heft 11 - Supplement: 61-79

53 Sachverständigenrat zur Begutachtung der gesamtwirtschaftlichen Entwicklung. (2002) a.a.O. und SVR KAiG. (1998) a.a.O.

54 Bundesgeschäftsstelle Qualitätssicherung gGmbH. (2005) a.a.O. und Hildebrandt GesundheitsConsult. IV-Datenbank Deutschland. http://www.gesundheitsconsult.de/db/2005.

55 Hildebrandt GesundheitsConsult GmbH (2004) a.a. O. und Neubauer G. Versorgungssteuerung über Vergütungsanreize: Braucht integrierte Versorgung integrierte Vergütung, in: Klauber J, Robra BP, Schellschmidt H (Hrsg.). Krankenhaus-Report 2005 - Wege zur Integration. Stuttgart New-York: Schattauer, 2005: 37-54

56 Siehe dazu beispielsweise das Praxisnetz Nürnberg. 